\title{
Analysis of Tear Glucose Concentration with Electrospray Ionization Mass Spectrometry
}

\author{
Christopher R. Taormina, Justin T. Baca, Sanford A. Asher, \\ and Joseph J. Grabowski \\ Department of Chemistry, University of Pittsburgh, Pittsburgh, Pennsylvania, USA \\ David N. Finegold \\ Department of Pediatrics, University of Pittsburgh School of Medicine, Pittsburgh, Pennsylvania, USA
}

We have developed a mass spectrometry-based method that allows one to accurately determine the glucose concentration of tear fluid. We used a $1 \mu \mathrm{L}$ micro-capillary to collect tear fluid from the tear meniscus with minimal irritation of the eye. We analyzed the $1 \mu \mathrm{L}$ volume of collected tear fluid with liquid-chromatography electrospray ionization mass spectrometry with the use of D-glucose- $6,6-\mathrm{d}_{2}$ as an internal standard. Repeated measurements and a recovery experiment on pooled, onion-induced tears showed that the analysis of the glucose in tears was precise ( $4 \%$ relative standard deviation) and provided $100 \%$ recovery. We found the tear glucose concentration of one fasting nondiabetic subject to be 13 to $51 \mu \mathrm{M}$ while the onion-induced tear glucose concentration of a different nondiabetic subject to be 211 to 256 $\mu$ M. (J Am Soc Mass Spectrom 2007, 18, 332-336) (C 2007 American Society for Mass Spectrometry

$\mathrm{I}$ n the United States, $9.6 \%$ of the population over $20 \mathrm{y}$ of age and $20.9 \%$ of the population over $60 \mathrm{y}$ of age have Diabetes mellitus [1]. The Diabetes Control and Complications Trial has shown that close monitoring and tight control of blood glucose concentration is critical in preventing the complications of diabetes [2]. Current self-monitoring methods rely on invasive finger sticks to directly measure blood glucose levels to provide the critical information required to achieve glycemic control. A number of noninvasive approaches to monitoring blood glucose concentrations are being pursued; however, none have been successfully developed to the point where they have gained widespread clinical acceptance [3]. There has been significant recent activity in exploring noninvasive monitoring by using tear fluid glucose as an indicator for blood glucose [4-6]. Such an approach will be effective only if the glucose concentrations in tear fluid are a reliable surrogate for blood glucose concentrations.

A survey of the literature over the last 70 years on tear glucose determinations indicates significant disagreement in measured tear glucose concentrations and

Published online November 2, 2006

Address reprint requests to J. J. Grabowski, University of Pittsburgh, Chevron Science Center, 219 Parkman Ave., Pittsburgh, PA 15260, USA. E-mail: joeg@pitt.edu. on the relationship of tear fluid glucose to blood glucose concentrations. Daum and Hill [7] noted in their 1982 review of the human tear glucose literature that reported values of tear glucose in normal individuals range between 0 and $3600 \mu \mathrm{M}$ with the median values between 110 and $280 \mu \mathrm{M}$. More recently, a study of six nondiabetic adults found glucose concentrations in tear fluid ranging between 128 and $166 \mu \mathrm{M}$ with an average of $139 \mu \mathrm{M}$ [8].

The differences in tear glucose concentrations between these reports are likely due to the use of different tear fluid collection methods. Van Haeringen and Glasius specifically addressed the dependence of tear fluid glucose on the method of collection [9]. They used a glucose dehydrogenase method to analyze chemically stimulated tears collected with a capillary and mechanically stimulated tear fluid collected by filter paper. Van Haeringen and Glasius found higher glucose concentrations in the tear fluid collected by filter paper and concluded that the increase is due to the mechanical stimulation of the corneal and conjunctival epithelium [9]. Other studies reported similar findings in experiments on rabbits [10] and in subjects who were tested immediately after swimming [7]. Therefore, it is apparent that to determine a physiologically relevant, baseline tear glucose concentration, tear fluid must be acquired with minimal tear stimulation and eye irritation. 
Several previous studies used mass spectrometry to analyze tears for constituents other than glucose. Fung et al. [11] characterized the protein profile in the human tear fluid with MALDI-ToF and electrospray ionization mass spectrometry. Ham et al. [12] identified the lipid profile in tear fluid and reported on their variations between normal and dry eyes. There have been many reports of glucose measurement by mass spectrometry on bodily fluids other than tears. The majority of these studies uses gas chromatography and an electron ionization source [13-16]. With gas chromatography, glucose typically needs to be derivatized to make it more volatile. Other studies used liquid chromatography to bypass the volatility issue [17, 18]. Sodium [19], lithium $[20,21]$, cesium [17], and chloride [22 $]^{\circ}$ ions $^{\circ}$ have ${ }^{\circ}$ been used with electrospray ionization to form the cationic adduct of glucose from aqueous solutions.

We discuss here a new, robust method of determining physiological tear glucose concentrations by using electrospray ionization mass spectrometry (ESI-MS). This method enables reliable studies of tear glucose concentrations in tear fluid. We use a tear fluid sampling method that is minimally irritating to the eye to collect $1 \mu \mathrm{L}$ tear samples. We validate the ESI-MS method by using glucose standard solutions, artificial tear fluid, and large volumes of stimulated tears. We determine tear glucose concentration in a nondiabetic, fasting adult.

\section{Experimental}

All tear samples were collected and transferred with 1 $\mu \mathrm{L}$ "Microcaps" micro-capillaries (Drummond Scientific Co., Broomall, PA). The tear film meniscus on the bottom eyelid was visualized using a slit lamp ophthalmic microscope (American Optics, Burlington, Canada). The micro capillary was gently touched to this tear fluid meniscus and the tear fluid was drawn in by capillary action. During half the collections, there was inadequate tear fluid to immediately fill the capillary with one touch. In these cases, the subject was asked to blink to redistribute the tear fluid and the capillary was again touched to the meniscus. This process was repeated until the capillary was completely filled. A few samples required as many as five repetitions of this process. Care was taken throughout tear fluid collection to avoid stimulation of reflex tearing. The aqueous glucose standards were collected and transferred with the same process as a tear sample; the capillary was touched to the solution surface and was filled by capillary action. For studies involving larger volumes of stimulated tears, the subject chopped onions until tearing was induced. These tears were collected by capillary action with glass Pasteur pipettes $(2 \mathrm{~mL})$ and pooled for each subject to analyze a homogeneous sample.

Immediately after collection, the tear sample was transferred to a glass vial (Agilent, Palo Alto, CA) with a glass insert (Restek, Bellafonte, PA) containing $10 \mu \mathrm{L}$ of acetonitrile (Mallinckrodt Baker, Inc., Phillipsburg,
$\mathrm{NJ})$ and $10 \mu \mathrm{L}$ of an aqueous stock solution of isotopically labeled glucose (D-glucose-6,6- $\mathrm{d}_{2}, 99 \%$ labeled, Cambridge Isotope Laboratories, Andover, MA) used as an internal standard. A typical value for the concentration of the D-glucose- $6,6-\mathrm{d}_{2}$ stock solution was 27.5 $\mu \mathrm{M}$, corrected for the $99 \%$ labeling (leading to a D-glucose-6,6- $\mathrm{d}_{2}$ concentration of $13.1 \mu \mathrm{M}$ in the sample injected into the mass spectrometer). The vials were then shaken and centrifuged for $5 \mathrm{~min}$ to ensure mixing and that the liquid was collected at the bottom of the vial. The samples were refrigerated at $5^{\circ} \mathrm{C}$ until analyzed.

Glucose concentrations were measured using ESIMS. Samples were analyzed by flow injection analysis on a HP 1000 LC-MS (Agilent). Initial studies used an autosampler connected directly to the mass spectrometer. However, our optimized method used a 2 $\mu \mathrm{m}$ filter and a Nova-Pak $\mathrm{C}_{18} 3.9 \times 150 \mathrm{~mm}$ column (Waters, Milford, MA) placed between the autosampler and the mass spectrometer. In both methods, the mobile phase consisted of acetonitrile and water with $0.1 \%$ formic acid (Mallinckrodt Baker, Inc.) with a constant flow rate of $0.2 \mathrm{~mL} / \mathrm{min}$. The ratio of water to acetonitrile was $1: 1$ in the absence of the column and 17:3 with the column. The ion chromatograms of sodiated glucose isotopes $(\mathrm{m} / z 203$ for natural glucose and $m / z 205$ for D-glucose-6,6- $\mathrm{d}_{2}$ ) were selected from the total ion chromatogram using the ChemStation software Rev. A.06.03-[509]. These selected ion chromatograms were integrated to provide peak areas for the two glucose signals. During integration, the software option "baseline hold" was used. The peak areas of $m / z 203$ and 205 were used to determine the glucose-to-D-glucose- $6,6-\mathrm{d}_{2}$ ratio. When calculating the peak area ratio, we took into account the $1.3 \%$ $m+2$ natural isotopic contribution of $\mathrm{Na}^{+}$(glucose), $m / z 203$, to $m / z 205 ; 1.3 \%$ of the observed glucose peak area of $\mathrm{m} / \mathrm{z} 203$ was subtracted from the observed $\mathrm{m} / \mathrm{z}$ 205 peak area to determine the area due solely to the internal standard. Each sample was injected three times at $6.5 \mu \mathrm{L}$ per injection and the average glucose concentration recorded. All human samples were collected through protocols approved by the University of Pittsburgh Institutional Review Board.

\section{Results and Discussion}

The cationization of glucose with $\mathrm{H}^{+}, \mathrm{Na}^{+}, \mathrm{K}^{+}$, or $\mathrm{Pb}^{2+}$ was examined. An initial survey comparing negative ion with positive ion ESI suggested greater promise with positive ion methods. With the exception of sodium, millimolar concentrations of the acid or salt were needed to observe the glucose cation adducts as the base peak. The sodium-glucose adduct was present in all of the spectra and, in the majority of them, was the most abundant ion. Since sodiated glucose dominated even without the addition of sodium and since tear fluid $^{\circ}$ contains $^{\circ} \sim 160^{\circ} \mathrm{mM}^{\circ}$ sodium $^{\circ}\left[23{ }^{\circ},{ }^{\circ}\right.$ our $^{\circ}$ glucose analysis is based on the sodiated glucose ion. Further 


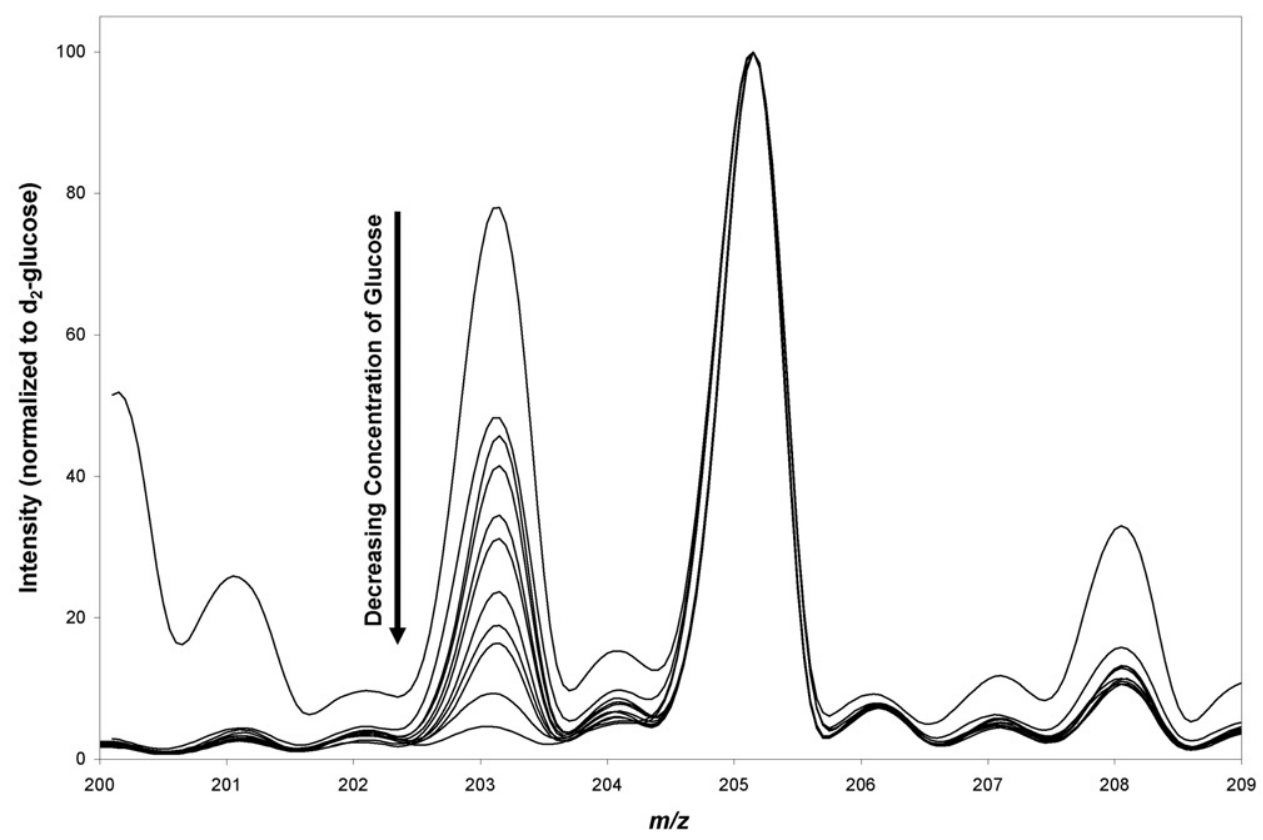

Figure 1. Eleven spectra of aqueous solutions of known glucose concentrations (0 to $200 \mu \mathrm{M})$ with intensities normalized to the internal standard D-glucose-6,6- $\mathrm{d}_{2}$ signal intensity at $m / z 205$. The linear regression of the experimental glucose concentration versus the known glucose concentration provided the best fit equation of $y=0.99 x-0.78\left(R^{2}=0.98\right)$.

experiments were conducted to ascertain the solvent composition (water, acetonitrile, and methanol) that provided the maximum signal for sodiated glucose. Based on the results of these experiments, a 1:1 ratio of water to acetonitrile for the sample solvent was selected. For the experiments without the use of the Nova-Pak $\mathrm{C}_{18}$ column, the 1:1 solvent ratio was also used for the mobile phase with the addition of $0.1 \%$ formic acid to the aqueous phase to produce more stable baseline ion currents. In our initial work, ${ }^{13} \mathrm{C}_{6}$ glucose was used as an internal standard but an unidentified peak at $\mathrm{m} / \mathrm{z} 209$ (which would interfere with the detection of sodiated ${ }^{13} \mathrm{C}_{6}$-glucose) was observed in the absence of the internal standard. Therefore, D-glucose- $6,6-\mathrm{d}_{2}$ was a superior alternative since no signal was observed at $\mathrm{m} / \mathrm{z} 205$ in the absence of the internal standard.

With the optimal solvents and internal standard established, the effect of the biological matrix on the glucose signal was examined. The sodiated glucose signal intensity observed for both artificial and real tear fluid was low compared with the background signal in our initial studies in which samples were directly infused into the mass spectrometer. Addition of a $\mathrm{C}_{18}$ column separated proteins, such as lysozyme, globulin, and albumin, and excess salts from the elution time of glucose. This in-line purification increased both the sensitivity and signal-to-noise ratio for sodiated glucose. Analysis of the blank (a protein-free, glucose-free artificial $^{\circ}$ tear $^{\circ}$ solution $^{\circ}[4]^{\circ}$ showed $^{\circ}$ an $^{\circ}$ average ${ }^{\circ}$ glucose concentration of $0.98 \pm 0.56 \mu \mathrm{M}$. With the use of the column the statistical limit of detection was lowered to
$3 \mu \mathrm{M}$. These improvements occurred in the presence of the column with the mobile phase optimized at a solvent ratio of $17: 3$ of water with $0.1 \%$ formic acid to acetonitrile which maximized the glucose signal and minimized overlapping peaks.

The precision of the microcapillaries was measured by gravimetric means. Five individual capillaries were weighed, filled with water at $20^{\circ} \mathrm{C}$, and weighed again. The average value for the water contained in the capillaries was $1.008 \pm 0.013 \mathrm{mg}(1.011 \pm 0.013 \mu \mathrm{L}$ at $20^{\circ} \mathrm{C}$ ). Therefore, in all of our analysis of tears we assume a collection volume of $1.00 \mu \mathrm{L}$.

To test the linearity of the method, aqueous solutions with known glucose concentrations ( 0 to $200 \mu \mathrm{M})$ were prepared and analyzed. The linear regression of measured versus added glucose gave an equation of $\mathrm{y}=$ $0.99 \times-0.78\left(R^{2}=0.98\right)$. The increase in the relative sodiated glucose signal $\mathrm{m} / \mathrm{z} 203$ can be easily seen when the mass spectra are normalized to the sodiated D-glucose- $6,6-\mathrm{d}_{2^{\circ}}$ signal $^{\circ} \mathrm{m} / \mathrm{z} 205^{\circ}$ (Figure ${ }^{\circ}$ ).

The mass spectral measurement over time of the glucose concentration in the 1:1 ratio of water-to-acetonitrile was examined. A $200 \mu \mathrm{M}$ glucose aqueous solution was prepared and sampled multiple times. These vials were stored in a refrigerator and tested immediately, a few $\mathrm{h}$ later, $3 \mathrm{~d}$ later, and $1 \mathrm{wk}$ later. The average value for all of the samples was $201 \pm 15 \mu \mathrm{M}$ with no increasing or decreasing trend over storage time (data not shown).

The reproducibility of the method was determined with the use of a pooled sample of onion-induced tears. Six samples were collected from the pool and analyzed. 


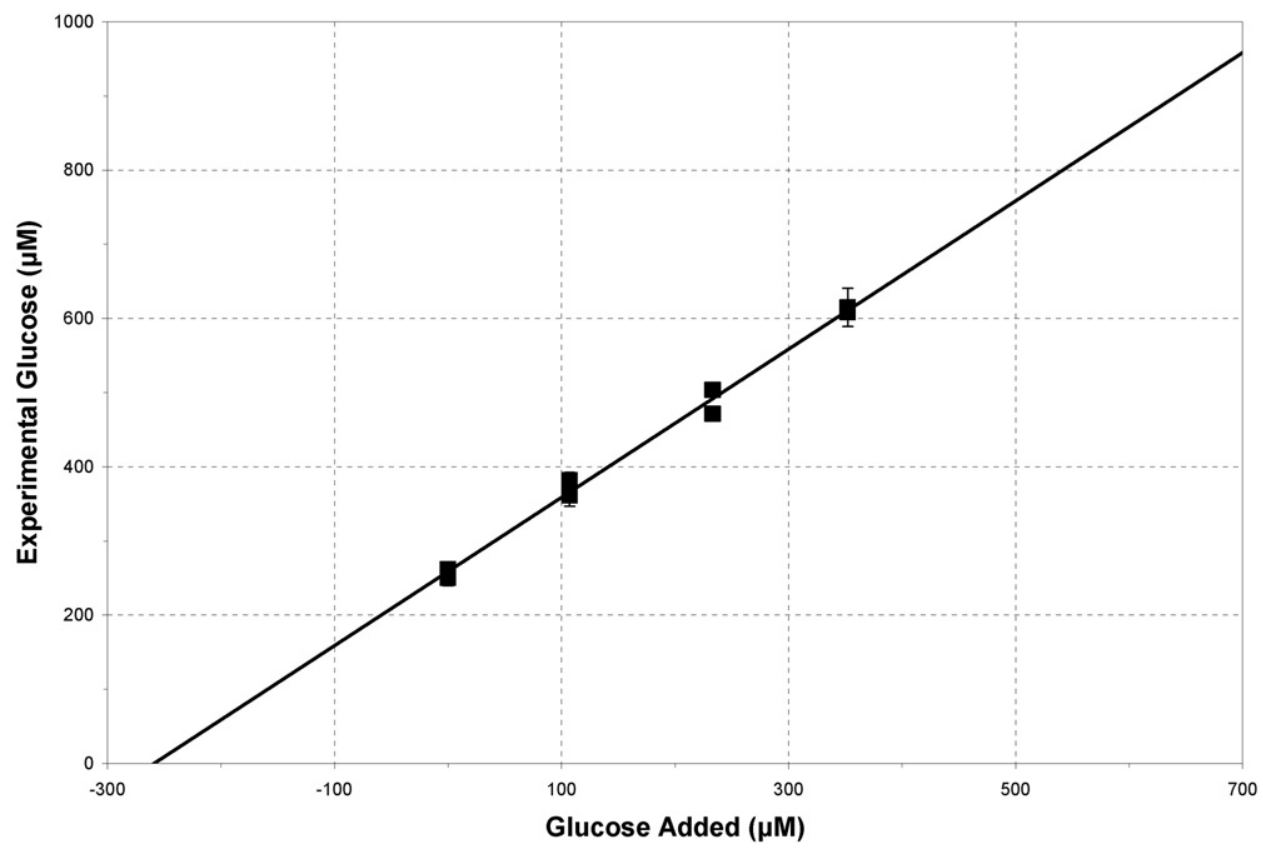

Figure 2. Duplicate samples of pooled, induced tears at four different concentrations of added glucose were analyzed. Error bars are \pm one standard deviation from the average value of each sample. Linear regression analysis gave an equation of $y=0.99 x+256\left(R^{2}=0.99\right)$ and a recovery range of 94 to $111 \%$ of the glucose spike.

The average glucose concentration was $211 \pm 8 \mu \mathrm{M}$ in this stimulated tear sample (data not shown).

A recovery experiment was performed on another pooled sample of onion-induced tears. The tears were spiked with aqueous glucose solutions to increase the glucose concentration by $0,107,233$, and $352 \mu \mathrm{M}$. Linear regression of measured versus added glucose gave an equation ${ }^{\circ}$ of $^{\circ} \mathrm{y}^{\circ}=0.99^{\circ} \mathrm{x}^{\circ}+{ }^{\circ} 256^{\circ}\left(\mathrm{R}^{2^{\circ}}=0.99\right)^{\circ}$ (Figure 2$){ }^{\circ}{ }^{\circ} \mathrm{We}$ also determined that the induced tear fluid contained $256 \mu \mathrm{M}$ glucose.

The average relative standard deviation over the three $6.5 \mu \mathrm{L}$ injections of the diluted tear samples studied with concentrations greater than $50 \mu \mathrm{M}$ glucose was $4 \%$. For the samples studied with concentrations less than $50 \mu \mathrm{M}$ glucose, the average relative standard deviation of the three measurements was $15 \%$. The FDA currently requires self-blood-glucose monitors to have an accuracy of within $15 \%$. Within one standard deviation, the calibration curve is indistinguishable from $y$ $=\mathrm{x}$ and the recovery experiment shows 100\% recovery of added glucose. We therefore feel confident using D-glucose- $-6,6-\mathrm{d}_{2}$ as the internal standard and the $y=x$ calibration curve to determine tear glucose concentrations.

We performed two studies on a nondiabetic subject who fasted overnight. During both sessions, which were separated by $1 \mathrm{wk}$, three samples were taken from each eye. Samples from the same eye were collected at $10 \mathrm{~min}$ intervals. The tear glucose concentration from this subject during the two sessions ranged from 13 to $51^{\circ} \mu \mathrm{M}^{\circ}$ with ${ }^{\circ}$ an $^{\circ}$ average ${ }^{\circ}$ of ${ }^{\circ} 32^{\circ} \mu \mathrm{M}^{\circ}$ (Table $\left.{ }^{\circ} 1\right) .^{\circ}$ The concentration of tear glucose determined in this work for this nondiabetic subject is about 4-fold less than the tear ${ }^{\circ}$ glucose $^{\circ}$ concentrations $^{\circ}$ recently $^{\circ}$ reported $^{\circ}$ [8]. Whereas previous studies used collection methods that either ${ }^{9}$ touched ${ }^{\text {the }}{ }^{\circ}$ eye ${ }^{\circ}$ or $^{\circ}$ chemically ${ }^{\circ}$ stimulated ${ }^{9}$ ears 97 , 9, 10], ${ }^{\circ}$ we $^{\circ}$ used $^{\circ}$ glass $^{\circ}$ micro-capillaries ${ }^{\circ}$ to ${ }^{\circ}$ collect $^{\circ}{ }^{\circ}$ single microliter volume of tear fluid from the tear meniscus without touching the eye. This decrease in reported tear glucose concentration is likely due to our less irritating method of tear collection. The onion-stimulated tears (from a different subject) showed a 7- to 8-fold higher glucose concentration than in the absence of stimulation.

\section{Conclusions}

In the present study, we demonstrate a robust ESI-MS method for determining tear glucose concentration in tear fluid. Liquid chromatography separates glucose from the majority of salts and proteins, such as lysozyme, globulin, and albumin, in tears reducing interference of the biological matrix on the sodiated-glucose

Table 1. Tear glucose concentration of a nondiabetic subject

\begin{tabular}{lccccc}
\hline & \multicolumn{2}{c}{ Session 1 } & & \multicolumn{2}{c}{ Session 2 } \\
\cline { 2 - 3 } \cline { 5 - 6 } Sample & Left eye & Right eye & & Left eye & Right eye \\
\hline \hline 1 & 22 & 38 & & 44 & 48 \\
2 & 51 & 37 & & 50 & 25 \\
3 & 13 & 17 & & 14 & 24 \\
\hline
\end{tabular}

Note: Concentrations are micromolar. 
signal. The resulting increase in the sensitivity and signal-to-noise ratio of glucose over data obtained without the column allows measurements of tear glucose concentrations as low as $10 \mu \mathrm{M}$ (limit of quantification) by relying on an internal standard. We use a $1 \mu \mathrm{L}$ tear sampling method that is minimally irritating to the eye. This method enables reliable studies of physiological tear glucose concentrations in nonstimulated tears. A preliminary study indicates that the physiological, baseline tear glucose concentration is substantially below previous reports. Further studies are warranted to determine the inter- and intra-individual variation in baseline tear glucose concentration. This method will be used to establish normal values for tear glucose concentration and can monitor changes in tear glucose with changes in blood glucose. Application of this LC-MS method can help us to understand tear glucose physiology. Our future work will determine whether monitoring tear glucose concentration is a viable approach for noninvasive blood glucose monitoring. We are now using this method for tear glucose concentration measurements to examine a population of nondiabetic and diabetic subjects to determine their tear fluid glucose concentrations and their correlations of tear glucose with blood glucose.

\section{Acknowledgments}

The authors thank Kasi Somayajula for his consultation. This research was supported of the National Institutes of Health grant DK-55348 to SAA.

\section{References}

1. National Diabetes Statistics; NIH Publication No. 06-3892. U.S. Department of Health and Human Services, National of Institutes of Health, National Institute of Diabetes, and Digestive and Kidney Diseases, National Diabetes Information Clearinghouse: Bethesda, MD, 2005.

2. Shamoon, H. D.; H.; Fleischer, N.; Engel, S.; Saenger, P.; Strelzyn, M.; Litwak, M.; Wylierosett, J. F. A.; and The Diabetes Control and Complications Trial Research Group. The Effect of Intensive Treatment of Diabetes on the Development and Progression of Long-Term Complications in Insulin-Dependent Diabetes Mellitus. New Engl. J. Med. 1993, 329, 977-986.

3. Khalil, O. S. Noninvasive Glucose Measurement Technologies: An Update from 1999 to the Dawn of the New Millennium. Diabetes Technol. Therapeut. 2004, 6, 660-697.

4. Alexeev, V. L.; Das, S.; Finegold, D. N.; Asher, S. A. Photonic Crystal Glucose-Sensing Material for Noninvasive Monitoring of Glucose in Tear Fluid. Clin. Chem. 2004, 50, 2353-2360.
5. Badugu, R.; Lakowicz, J. R.; Geddes, C. D. Ophthalmic Glucose Sensing: A Novel Monosaccharide Sensing Disposable and Colorless Contact Lens. Analyst (Cambridge, UK) 2004, 129, 516-521.

6. March, W. F.; Mueller, A.; Herbrechtsmeier, P. Clinical Trial of a Noninvasive Contact Lens Glucose Sensor. Diabetes Technol. Therapeut. 2004, 6, 782-789.

7. Daum, K. M.; Hill, R. M. Human Tear Glucose. Invest. Ophthalmol. Vis. Sci. 1982, 22, 509-514.

8. Chen, R.; Jin, Z.; Colon, L. A. Analysis of Tear Fluid by CE/LIF: A Noninvasive Approach for Glucose Monitoring. J. Capillary Electrophoresis 1996, 3, 243-248.

9. Van Haeringen, N. J.; Glasius, E. Collection Method-Dependent Concentrations of Some Metabolites in Human Tear Fluid, with Special Reference to Glucose in Hyperglycemic Conditions. Albrecht von Graefes Archiv. Klin. Experim. Ophthalmol. 1977, 202, 1-7.

10. Kilp, H.; Heisig, B. Changes of the Glucose and Lactate Concentration in Tears after Mechanical Alteration and Contact Lens Wearing. Albrecht von Graefes Archi. Klin. Experim. Ophthalmol. 1975, 193, 259-267.

11. Fung, K. Y. C.; Morris, C.; Sathe, S.; Sack, R.; Duncan, M. W. Characterization of the in vivo Forms of Lacrimal-Specific Proline-Rich Proteins in Human Tear Fluid. Proteomics 2004, 4, 3953-3959.

12. Ham, B. M. Jacob, J. T. Keese, M. M. Cole, R. B. Identification, Quantification, and Comparison of Major Nonpolar Lipids in Normal and Dry Eye Tear Lipidomes by Electrospray Tandem Mass Spectrometry. J. Mass Spectrom. 2004, 39, 1321-1336.

13. Clapperton, A. T.; Coward, W. A.; Bluck, L. J. C. Measurement of Insulin Sensitivity Indices Using 13C-Glucose and Gas Chromatography/Combustion/Isotope Ratio Mass Spectrometry. Rapid Commun. Mass Spectrom. 2002, 16, 2009-2014.

14. Dube, G.; Henrion, A.; Ohlendorf, R.; Vidal, C. Application of the Combination of Isotope Ratio Monitoring with Isotope Dilution Mass Spectrometry to the Determination of Glucose in Serum. Rapid Commun. Mass Spectrom. 2001, 15, 1322-1326.

15. Lee, J.; Chung, B. C. Simultaneous Measurement of Urinary Polyols Using Gas Chromatography/Mass Spectrometry. J. Chromatogr. B Anal. Technol. Biomed. Life Sci. 2006, 831, 126-131.

16. Di Gioia, M. L.; Leggio, A.; Le Pera, A.; Liguori, A.; Napoli, A.; Siciliano, C.; Sindona, G. Quantitative Analysis of Human Salivary Glucose by Gas Chromatography-Mass Spectrometry. J. Chromatogr. B Anal. Technol. Biomed. Life Sci. 2004, 801, 355-358.

17. Rogatsky, E.; Jayatillake, H.; Goswami, G.; Tomuta, V.; Stein, D. Sensitive LC MS Quantitative Analysis of Carbohydrates by Cs+ Attachment. J. Am. Soc. Mass Spectrom. 2005, 16, 1805-1811.

18. Wan, E. C. H.; Yu, J. Z. Determination of Sugar Compounds in Atmospheric Aerosols by Liquid Chromatography Combined with Positive Electrospray Ionization Mass Spectrometry. J. Chromatogr. A 2006, 1107, 175-181.

19. McIntosh, T. S.; Davis, H. M.; Matthews, D. E. A Liquid Chromatography-Mass Spectrometry Method to Measure Stable Isotopic Tracer Enrichments of Glycerol and Glucose in Human Serum. Anal. Biochem. 2002, 300, 163-169.

20. Bruggink, C.; Maurer, R.; Herrmann, H.; Cavalli, S.; Hoefler, F. Analysis of Carbohydrates by Anion Exchange Chromatography and Mass Spectrometry. J. Chromatogr. A 2005, 1085, 104-109.

21. Guignard, C.; Jouve, L.; Bogeat-Triboulot, M. B.; Dreyer, E.; Hausman J.-F.; Hoffmann, L. Analysis of Carbohydrates in Plants by HighPerformance Anion-Exchange Chromatography Coupled with Electrospray Mass Spectrometry. J. Chromatogr. A 2005, 1085, 137-142.

22. Zhu, J.; Cole, R. B. Ranking of Gas-Phase Acidities and Chloride Affinities of Monosaccharides and Linkage Specificity in CollisionInduced Decompositions of Negative Ion Electrospray-Generated Chloride Adducts of Oligosaccharides. J. Am. Soc. Mass Spectrom. 2001, 12, 1193-1204.

23. Gilbard, J. P. Human Tear Film Electrolyte Concentrations in Health and Dry-Eye Disease. Int. Ophthalmol. Clin. 1994, 34, 27-36. 